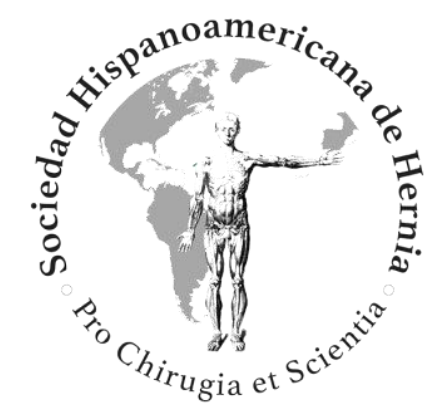

Punto de corte del nervio iliohipogástrico en la triple neurectomía por dolor inguinal crónico posoperatorio

\title{
Cutting point of the iliohypogastric nerve in the triple neuroctomy due to chronic inguinodynia
}




\section{OR 480}

Punto de corte del nervio iliohipogástrico en la triple neurectomía por dolor inguinal crónico posoperatorio

Cutting point of the iliohypogastric nerve in the triple neuroctomy due to chronic inguinodynia

Alfredo Moreno-Egea

Clínica Hernia. Hospital Universitario La Vega. Murcia (España)

Recibido: 05-11-2021

Aceptado: 05-11-2021

Autor para correspondencia: Alfredo Moreno-Egea. Clínica Hernia. Hospital Universitario La Vega. C/ Dr. Román Alberca, s/n. 3008 Murcia (España)

Correo electrónico: morenoegeaalfredo@gmail.com

DOI: $10.20960 /$ rhh. 00480

Conflictos de interés: el autor declara no tener conflictos de interés.

\section{RESUMEN}

La complicación más temible e invalidante para el paciente en una hernioplastia inguinal es el dolor inguinal crónico posoperatorio. La técnica de triple neurectomía se acepta como una opción cuando otras alternativas de tratamiento conservador (médicas 0 radiológicas) han fallado.

Palabras clave: Nervio iliohipogástrico, triple neurectomía, dolor inguinal crónico. 


\section{ABSTRACT}

The most redoubtable and invalidating complication for an inguinal hernioplasty patient is to suffer chronic inguinodynia. The triple neurectomy technique is accepted as an option when other conservative alternatives (either medical or radiological) have failed.

Keywords: Iliohypogastric nerve, triple neurectomy, chronic inguinodynia.

\section{INTRODUCCIÓN}

La complicación más temible e invalidante para el paciente en una hernioplastia inguinal es el dolor inguinal crónico posoperatorio. La técnica de triple neurectomía se acepta como una opción cuando otras alternativas de tratamiento conservador (médicas 0 radiológicas) han fallado ${ }^{1,2}$.

En la descripción «clásica» de esta técnica se considera como punto de corte para el nervio iliohipogástrico (e ilioinguinal) el que está localizado «sobre el músculo cuadrado lumbar»³. Esta afirmación es habitualmente imposible de realizar si se sigue literalmente la descripción publicada, ya que el trayecto de este nervio discurre lateralmente al borde externo de dicho músculo sobre el transverso abdominal hasta su penetración. En la mayoría de las ocasiones, solo el nervio ilioinguinal presenta un trayecto sobre el músculo cuadrado lumbar ${ }^{4-6}$. Así, el punto clásico de corte $(« C »)$ neural descrito es proximal y craneal en el espacio extraperitoneal y al seccionarse deja sin inervación a los músculos laterales de la pared abdominal, lo que causa una pseudohernia (figs. 1 y 2 A). Esta complicación se ha demostrado entre un 30 y $100 \%$ de los casos, según las variantes neurales, tras la triple neurectomía clásica ${ }^{7}$.

\section{PROPUESTA}


Para evitar este problema, proponemos como nuevo punto de corte uno que llamaremos «A» (anatómico), que está localizado a nivel intermuscular cerca de la cresta ilíaca (Tabla I). Este hecho tiene dos implicaciones quirúrgicas:

1. Reemplaza la necesidad de realizar una disección de la tradicional dirección proximal, cuyo fin es identificar el cuerpo muscular del cuadrado lumbar antes de proceder a la sección neural, por una disección distal en dirección a la cresta ilíaca, donde ambos nervios quedan a menor distancia. Este hecho evita entrar en conflicto con la zona grasa que rodea el músculo y supone un menor riesgo de sangrado.

2. Respeta las ramas musculares del nervio en el espacio extraperitoneal. Este punto de sección distal garantiza la inervación de la pared abdominal posterolateral, lo que evita la debilidad por denervación y la pseudonernia.

\section{MANIOBRA}

El nivel de entrada del nervio bajo su fascia se identifica en el cabestrillo muscular del transverso abdominal y se diseca separando las fibras musculares sin romperlas en dirección a la espina ilíaca posterosuperior. Las fibras musculares solo se separan fácilmente si dejamos ver el trayecto intermuscular del nervio, la salida de la rama posterior o glútea y las ramas musculares en un número medio de 46.

\section{DEMOSTRACIÓN EXPERIMENTAL}

Este planteamiento se ha verificado en un estudio con un cadáver y después se ha llevado a quirófano para valorar su viabilidad. Solo cuando se haya comprobado su viabilidad técnica sin aumentar la morbilidad por sangrado o rotura muscular, se plantea esta variante técnica para disminuir al mínimo la posibilidad de pseudohernia. Esto se debe a que, al seccionar el nervio a nivel intermuscular sobre la cresta ilíaca, después de dar la mayoría de sus ramas musculares, 
asegura la inervación de la pared abdominal posterolateral (figs. 2B y 3A-D).

Como consejo cabe mencionar que es conveniente evitar la sección del iliohipogástrico si no se demuestra su afectación en el mapeo (doble, no triple neurectomía).

\section{CONCLUSIÓN}

La modificación del punto de corte clásico («C») en la triple neurectomía por dolor inguinal crónico posoperatorio por el nuevo punto anatómico «A» supone una ventaja clínica para el paciente y evita la aparición de una pseudohernia.

La operación de triple neurectomía por abordaje laparoscópico queda así constituida por tres teorías que le dan sustento anatómico, quirúrgico y científico-técnico:

1. La teoría de identificación o de los espacios triangulares retrolumbar y retroilíaco, que evita las lesiones neurales inadvertidas.

2. La teoría de la disección o fascial, que evita las lesiones vasculares.

3. La teoría de sección o de punto de corte neural, que evita la pseudohernia de la pared abdominal.

Con estas tres teorías completamos la descripción del proceso para su realización segura en clínica.

\section{BIBLIOGRAFÍA}

1. Moreno-Egea A. Surgical management of postoperative chronic inguinodynia by laparoscopic transabdominal preperitoneal approach. Surg Endosc. 2016;30(12):5222-7. DOI: 10.1007/s00464-016-4867-0

2. Moreno-Egea A, Mayagoitia JC. Guía clínica para el manejo del dolor inguinal crónico posoperatorio. Rev Hispanoam Hernia. 2021;9(2):1316. DOI: $10.20960 /$ rhh.00448 
3. Chen DC, Hiatt JR, Amid PK. Operative management of refractory neuropathic inguinodynia by a laparoscopic retroperitoneal approach. JAMA Surg. 2013;148(10):962-7. DOI: 10.1001/jamasurg.2013.3189

4. Moreno-Egea A. Anatomía quirúrgica para especialistas en pared abdominal. Atlas fotográfico de disección. Valencia: Ed. SOHAH; 2021. 5. Moreno-Egea A. Espacio retrolumboilíaco quirúrgico: estudio anatómico e implicaciones en el abordaje laparoscópico del dolor inguinal crónico. Rev Hispanoam Hernia. 2020;8(1):25-32. DOI: 10.20960/rhh.00222

6. Moreno-Egea A. A study to improve identification of the retroperitoneal course of iliohypogastric, ilioinguinal, femorocutaneous and genitofemoral nerves during laparoscopic triple neurectomy. Surg Endosc. 2021;35(3):1116-25. DOI: 10.1007/s00464020-07476-w

7. Moore AM, Bjurstrom MF, Hiatt JR, Amid PK, Chen DC. Efficacy of retroperitoneal triple neurectomy for refractory neuropathic inguinodynia. Am J Surg. 2016;212(6):1126-32. DOI: 10.1016/j.amjsurg.2016.09.012

Tabla I. Reformulación del punto de corte neural para el nervio iliohipogástrico en la operación de triple neurectomía

\begin{tabular}{|l|l|l|}
\hline & $\begin{array}{l}\text { Punto } \\
\text { clásico }\end{array}$ & $\begin{array}{l}\text { Punto } \\
\text { anatómico }\end{array}$ \\
\hline Escuela & D. Chen & A. Moreno-Egea \\
\hline Disección & Proximal & Distal \\
\hline Pared abdominal & Posterior & Anterior \\
\hline Sobre músculo & $\begin{array}{l}\text { Cuadrado } \\
\text { lumbar }\end{array}$ & $\begin{array}{l}\text { Transverso } \\
\text { Nivel de cresta } \\
\text { ilíaca }\end{array}$ \\
\hline $\begin{array}{l}\text { Preserva rama ilíaca } \\
\text { perforante } \\
\text { musculares }\end{array}$ & No & Sí \\
\hline Pseudohernia & $30-100 \%$ & $0 \%$ \\
\hline
\end{tabular}




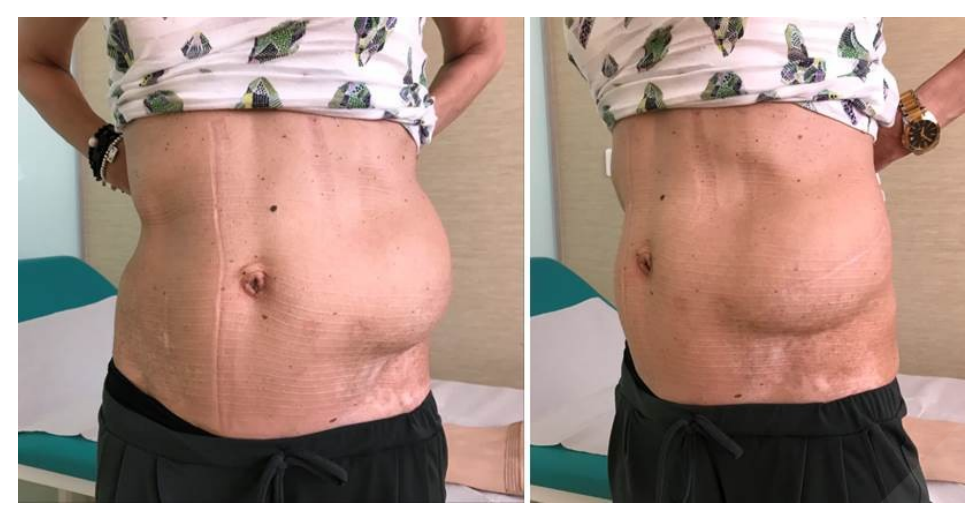

Figura 1. Paciente con pseudohernia por denervación tras corte del nervio iliohipogástrico en una nefrectomía izquierda.

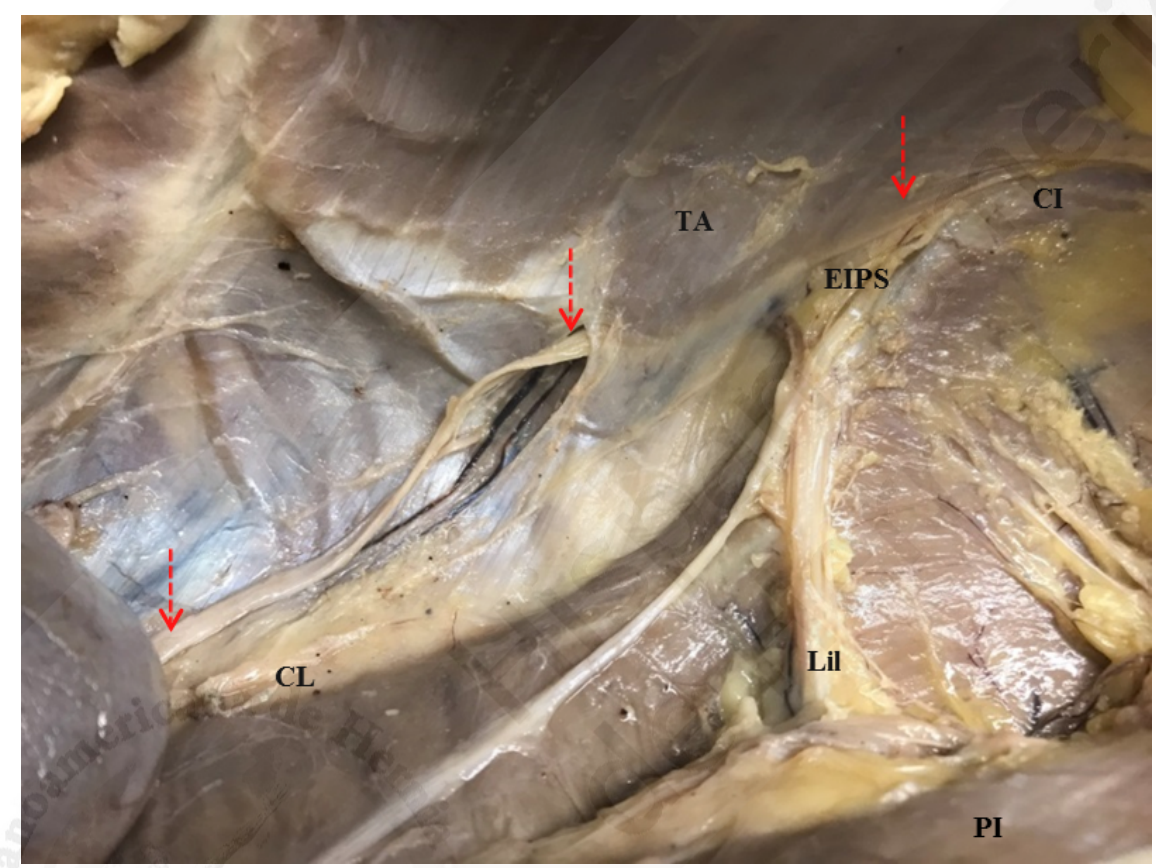

Figura 2A. Trayecto fascial desde el borde lateral del CL hasta el cabestrillo muscular del TA. Trayecto intermuscular hasta la EIPS y cresta ilíaca, acercándose al II.

TA: músculo transverso abdominal; $\mathrm{Cl}$ : cresta ilíaca; $\mathrm{CL}$ : cuadrado lumbar; PI: psoasilíaco; Lil: ligamento iliolumbar; EIPS: espina ilíaca posterosuperior. 


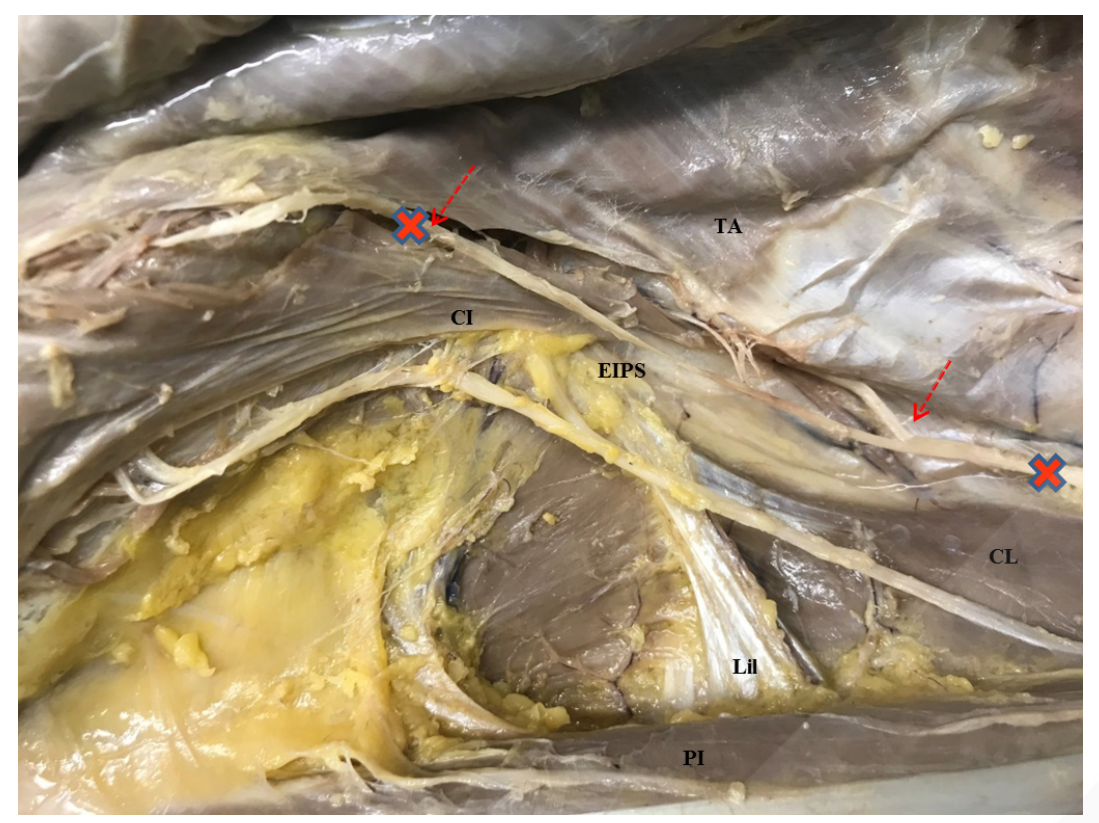

Figura 2B. Nervio en el espacio extraperitoneal con los puntos de corte clásico y anatómico para dejar las ramas musculares indemnes para evitar la pseudohernia. Las flechas indican el trayecto del nervio iliohipogástrico.

TA: músculo transverso abdominal; $\mathrm{Cl}$ : cresta ilíaca; $\mathrm{CL}$ : cuadrado lumbar; PI: psoasilíaco; Lil: ligamento iliolumbar; EIPS: espina ilíaca posterosuperior.
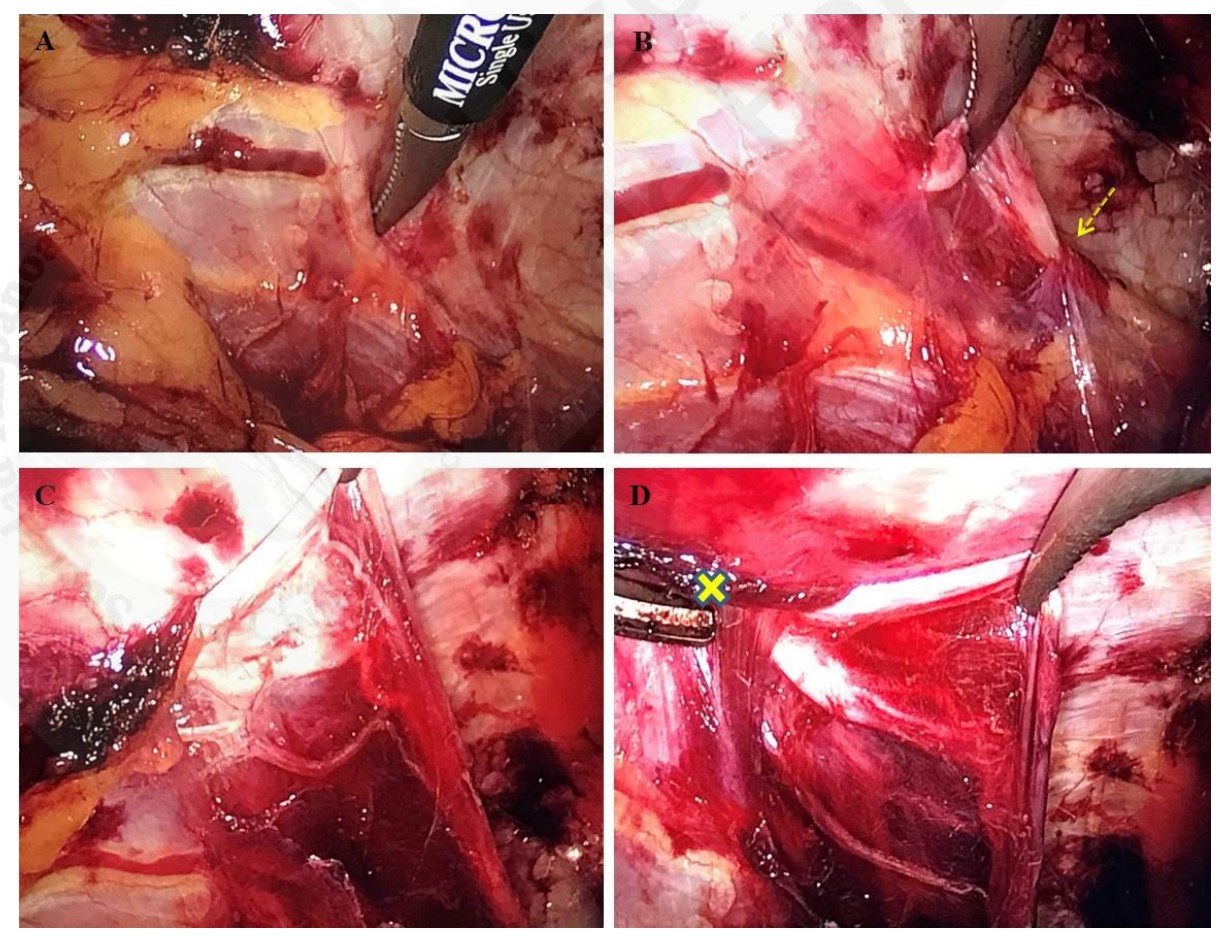
Figura 3. Aplicación de la técnica con nuevo punto de corte para el nervio iliohipogástrico. A. Identificación neural por presión sobre el transverso. B. Liberación fascial sobre el cabestrillo muscular. C. Disección distal intermuscular para liberar las ramas musculares. D. Sección distal sobre cresta ilíaca. 